\title{
La fragilidad de la memoria: creencias falsas y memoria autobiográfica, una revisión preliminar*
}

Fragility in Memory: False Beliefs and Autobiographical Memory, a Preliminary Review

Recepción: 27 Octubre 2016 | Aceptación: 30 Octubre 2017

\author{
Joselyne Nájera Fernández \\ Universidad de Costa Rica, Costa Rica \\ ORCID: http://orcid.org/0000-0001-7935-9689 \\ Mónica Salazar Villanea \\ Universidad de Costa Rica, Costa Rica \\ ORCID: http://orcid.org/0000-0001-9311-8195 \\ Jaime Fornaguera Trías \\ Universidad de Costa Rica, Costa Rica \\ ORCID: http://orcid.org/0000-0002-8833-8123
}

\footnotetext{
a Autor de correspondencia. Correo electrónico: jnajera@daad-alumni.de
}

Para citar este artículo: Nájera Fernández, J., Salazar Villanea, M., \& Fornaguera Trías, J. (2018). La fragilidad de la memoria: creencias falsas y memoria autobiográfica, una revisión preliminar. Universitas Psychologica, 17(4), 1-11. https://doi.org/10.11144/ Javeriana.upsy17-4.fmcf

\begin{abstract}
RESUMEN
La memoria es un proceso reconstructivo, donde distintos recuerdos buscan conformar una narrativa autobiográfica coherente. No obstante, dichos recuerdos no son completamente precisos, ya que en diversas investigaciones se ha encontrado que las personas tienden a reportar episodios de su pasado que sucedieron ya sea de manera distinta o que en realidad nunca ocurrieron. Tras considerar a la memoria autobiográfica como el mayor logro ontogenético humano, surge la interrogante respecto a cómo puede suceder un fenómeno tan impreciso como el de las memorias falsas. En el presente estudio teórico se revisa la principal evidencia científica respecto a la existencia de memorias falsas, se analiza su etiología y se profundiza en su posible rol adaptativo.

Palabras clave

memoria autobiográfica; memorias falsas; creencias falsas; sugestión.
\end{abstract}

\begin{abstract}
Memory is a reconstructive process in which different memories seek to form a coherent autobiographical narrative. Still, these memories seem to be not entirely accurate, since years of research have found that people tend to recall their past events differently from what happened, or even mention memories of events that did not occur. After considering autobiographical memory as the greatest human ontogenetic achievement, the question concerning the existence of such an imprecise phenomenon, as it is that of false memories, arises. The present theoretical study reviews the main scientific evidence regarding the existence of false memories, discusses its etiology and delves into its potential adaptive role. Keywords autobiographical memory; false memories: false beliefs; suggestibility.
\end{abstract}


La existencia del ser humano se encuentra fuertemente ligada a su memoria, puesto que a partir de esta se pueden integrar múltiples recuerdos y utilizarlos para el planeamiento del futuro, la resolución de problemas en el presente y el aprendizaje, por lo que se le atribuye un rol adaptativo (Hering, 1895). La memoria como tal, no es un constructo unitario, sino que se compone de varios sistemas diferenciados tanto filogenéticamente como ontogenéticamente (Staniloiu \& Markowitsch, 2012). Uno de estos sistemas es el de la memoria autobiográfica, entendida como la capacidad mnémica relacionada con los eventos y experiencias del sí mismo (Koriat, Goldsmith, \& Pansky, 2000), al entrelazar aspectos relativos al sí mismo, la motivación, la emoción y el significado personal atribuido a la experiencia vivida (Salazar, 2008). La memoria autobiográfica se diferencia de la memoria episódica (relacionada con eventos y experiencias) en que la información es específica, personal, duradera y significativa para el sí mismo, puesto que llega a conformar la historia de vida personal (Nelson, 1993).

Actualmente, se reconoce que la memoria humana no es una reproducción exacta de experiencias pasadas, sino más bien un "proceso imperfecto" que presenta varios tipos de errores y distorsiones (Schacter, Guerin, \& St. Jacques, 2011), inclusive en recuerdos de tipo autobiográfico (Staniloiu \& Markowitsch, 2012). No obstante, todavía no existe un marco unificado que brinde una definición operacional exacta sobre las memorias autobiográficas falsas, al ser un proceso complejo que involucra procedimientos cognitivos evaluativos de alto nivel (Brewin \& Andrews, 2017).

La presente revisión preliminar bibliográfica pretende indagar cuáles son los hallazgos más importantes respecto al fenómeno de las memorias autobiográficas falsas, y a partir de ello explorar su posible rol adaptativo en esta capacidad mnémica que resulta ser tan influyente en la construcción de la identidad (Wilson \& Ross, 2003).

\section{Memorias y creencias autobiográficas falsas}

El sistema de memoria del sí mismo (SMS) es el marco conceptual que enfatiza la relación recíproca que existe entre el sí mismo y la memoria, a partir de la concepción de que la percepción que tiene un individuo de sí mismo es intrínseca a la memoria de su vida (Prebble, 2014). De acuerdo con Conway (2005), el SMS consta esencialmente de dos componentes: el sí mismo operativo y la base de conocimiento de la memoria autobiográfica.

Las memorias autobiográficas son construcciones mentales transitorias y dinámicas que se generan a partir de una base de conocimiento subyacente, la cual contiene tres niveles de especificidad: i) períodos de vida, donde se representan lugares, personas, actividades, sentimientos y objetivos comunes de un período en particular (e. g., "las vacaciones familiares cuando tenía 12 años”); ii) eventos generales, que resultan ser más específicos que los períodos de vida y engloban representaciones de eventos repetidos o una secuencia de eventos relacionados (e. g., "estuvimos en la playa la mayor parte del tiempo") y iii) conocimiento específico sobre eventos, que implica información detallada vívidamente respecto a eventos individuales, comúnmente en la forma de imágenes visuales y atributos sensorio-perceptivos, los cuales tienden a desvanecerse rápidamente, a menos que sean recuerdos que marquen el inicio, redirijan el curso o reafirmen un aspecto relevante para el individuo (e. g., "conocí a mi mejor amigo mientras trataba de pescar un pez grande").

A la hora de recordar, se sigue un orden jerárquico que va de lo general a lo específico, cuando la persona debe comenzar por recordar el período de vida donde se ubica espacial y temporalmente el recuerdo, seguidamente los eventos generales que sucedieron durante dicha época y finalmente aquellos detalles específicos que caracterizan al recuerdo que se desea evocar (Schacter, 1996).

Por su lado, el segundo componente del SMS denominado el sí mismo operativo, es un 
mecanismo de funcionamiento ejecutivo que controla el acceso a la base del conocimiento y que se conduce a partir de las metas actuales establecidas por el individuo (Conway $\&$ Pleydell-Pearce, 2000). De esta manera, se mantiene una relación recíproca entre ambos durante la construcción del recuerdo, puesto que el sí mismo operativo controla la base del conocimiento a partir de la información que esta le brinda sobre los aspectos asociados con las metas del sí mismo.

Cabe mencionar, que el recordar eventos del pasado implica un proceso constructivo en el que se dan la re-transcripción, re-categorización e inclusive invención de la información que se encuentra almacenada (Staniloiu \& Markowitsch, 2012). Las reminiscencias van a variar dependiendo de cuáles son las metas del sí mismo en un momento dado (i. e., estado de humor, motivación, circunstancias, etc.) (Blank, 1998). De esta manera, cada vez que recordamos, construimos la información del evento con base en las condiciones actuales del sí mismo y, al mismo tiempo, esto conlleva a que esa nueva evocación sea re-almacenada e incorporada a la base del conocimiento sobre el evento (Tulving \& Markowitsch, 1998).

Lo anterior demuestra que, a pesar de que el recordar eventos del pasado por lo general se acompaña de un sentido de veracidad, donde el sujeto tiene el sentimiento subjetivo de que se están recuperando fragmentos de un episodio tal y como sucedió, la recuperación de la información implica cierto grado de imprecisión en el que las experiencias que se reconstruyen no son exactamente iguales a cómo sucedieron (Newman \& Lindsay, 2009). Lo anterior probablemente explica, en buena proporción, el por qué suelen haber errores menores como el confundir detalles sobre un evento o recordar eventos de manera distinta, dependiendo de la etapa de vida o estado emocional en el que se encuentre el sujeto.

Para los propósitos de la presente investigación, nace el interés respecto de cómo surgen imprecisiones mnémicas con implicaciones mayores, como el caso de recuerdos autobiográficos sobre eventos que en realidad nunca sucedieron (Schacter, 2001). A este tipo de memorias se les denomina memorias falsas, e involucran recuerdos autobiográficos, con niveles de representación tanto semánticos como episódicos $^{1}$, que no ocurrieron y son fuertemente creídos como ciertos a pesar de ser factualmente incorrectos (Mendez \& Fras, 2011).

A diferencia de las confabulaciones, las memorias falsas no se encuentran asociadas con un tipo de trastorno neurológico o trauma (Berlyne, 1972). Por su parte, Newman y Lindsay (2009) establecen que a partir de procesos como sugestión e imaginación es que se generan creencias falsas que dan base a la formación de memorias falsas. La existencia de las memorias falsas se puede atribuir a lo que Schacter (2001) plantea como los siete pecados de la memoria, los cuales describen cómo las operaciones mentales normales pueden llegar a ocasionar experiencias mnémicas imprecisas. La tabla 1 presenta una breve descripción de los mismos.

\section{Tabla 1}

Los siete pecados de la memoria

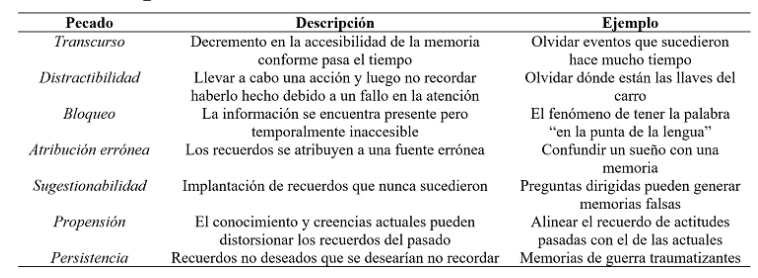

Fuente: elaboración propia, basada en Schacter, Chiao y Mitchell (2003, p. 228).

Dentro de la propuesta de Schacter (2001), llama la atención el rol de la sugestión en la formación de memorias falsas, puesto que a partir de ello se genera la creencia en el individuo de que un evento significativo sucedió y consecuentemente la imaginación comienza a construir una serie de recuerdos que den sentido a esa creencia (Mazzoni \& Memon, 2003). De esta manera, los procesos mnémicos buscan adecuarse a nuestras creencias y expectativas para poder cumplir con los schematas, o estructuras de conocimiento genéricas que describen los elementos esperados para ciertos tipos de situaciones (Bartlett, 1932). 
Dicho en otras palabras, si un sujeto incorpora a su narrativa autobiográfica la creencia de un evento falso con la premisa de que en verdad ocurrió, va a tender a imaginar los eventos o experiencias relacionadas con lo anterior, y estos van a buscar tener un sentido coherente en relación con lo que establece el guión del schemata. Sobre lo anterior, resulta importante resaltar lo poderosa que puede ser una creencia en la generación de memorias falsas, dado que, si en principio los procesos mnémicos buscan adecuarse a nuestras creencias y expectativas, es probable que su distorsión conlleve a la formación de recuerdos distorsionados y por ende a una narrativa de historia de vida distorsionada.

Numerosos estudios han demostrado que es factible modificar detalles sobre recuerdos de eventos pasados, e inclusive implantar recuerdos autobiográficos falsos sobre situaciones altamente inverosímiles (Finkelstein, 2017; Loftus \& Davis, 2006; Sherman, Follows, Mushore, Hampson-Jones, \& Wright-Evans, 2015).

Uno de ellos es el clásico estudio de Loftus y Pickrell (1995), donde se pidió a los padres de los sujetos experimentales que describieran tres eventos que ocurrieron durante la infancia de sus hijos. Posteriormente, los sujetos leían cuatro eventos proporcionados por sus padres (tres eran verdaderos y uno era falso), y se les pedía que recordaran información relacionada con dichas experiencias. Se encontró, tras varias entrevistas sucesivas con los sujetos, que un $25 \%$ del total en verdad creía que el recuerdo falso había sucedido (en este caso era el haberse perdido en el centro comercial de niños, que según sus padres en realidad nunca ocurrió).

Wade, Garry, Read y Lindsay (2002) adaptaron el estudio anterior reemplazando el uso de narrativas por fotografías, asumiendo que las personas tradicionalmente consideran que captan verazmente los eventos del pasado. Le mostraron a cada sujeto experimental cuatro fotografías, de las cuales tres eran reales y una falsa (aparece el sujeto en un globo aerostático junto con un familiar). En este caso, hasta 50 $\%$ de los sujetos recordó algo relativo al viaje en aerostático con un gran nivel de detalle. Los autores señalan que lo anterior puede deberse al componente episódico de familiaridad, en el que los sujetos elaboran recuerdos falsos producto de la cercanía del estímulo con eventos anteriores que sí sucedieron.

De acuerdo con Garry y Gerrie (2005), las fotografías ayudan a los sujetos a imaginar detalles sobre un evento que luego tienden a ser confundidos con la realidad, considerando a la imaginación como una fuente importante de influencia sobre la memoria. Lo importante no es si la fotografía es verdadera o falsa, ya que existen estudios como el de Lindsay, Hagen, Read, Wade y Garry (2004), en el que se demuestra cómo imágenes verdaderas pueden inclusive cultivar creencias falsas, sino el cómo a partir de una creencia falsa, la imaginación puede conducir a la evocación de múltiples recuerdos falsos.

Por otro lado, Laney, Morris, Bernstein, Wakefield y Loftus (2008) exploran el fenómeno de la sugestión, al brindar a los sujetos participantes memorias falsas sobre cómo en la niñez les gustaban los espárragos. Se encontró que en relación con el grupo control, los del grupo experimental estaban más seguros de que les habían gustado los espárragos desde la primera vez que los probaron, y se evidenció un repentino mayor agrado hacia dicho alimento, inclusive llegando los sujetos a pedirlo con mayor frecuencia en restaurantes y supermercados. En un estudio posterior, se retoma el experimento de los espárragos y señala que dicho efecto puede tener consecuencias a largo plazo respecto a los hábitos alimenticios, al demostrarse su persistencia inclusive hasta dos semanas después (Laney, Bowman-Fowler, Nelson, Bernstein \& Loftus, 2008).

Lo anterior arroja la interrogante respecto a cómo la publicidad puede llegar a generar un efecto similar al utilizar la sugestión como un medio para crear falsas creencias en el individuo que conduzcan a un mayor consumo de los productos. Braun, Ellis y Loftus (2002) exploraron el tema a través de dos experimentos. En el primero, los participantes vieron un anuncio de Disney que sugería que le habían estrechado la mano a Mickey Mouse, y se descubrió que, en comparación con el grupo 
control, los sujetos del grupo experimental tenían una mayor certeza de que dicho evento en verdad había sucedido, ya sea por familiaridad con un recuerdo verdadero o por la imaginación de un recuerdo falso. En el segundo experimento, los participantes observaron un anuncio en el que se sugería que le habían estrechado a Bugs Bunny (evento inverosímil), y se encontró que en comparación con el grupo control, los sujetos del grupo experimental presentaban una mayor certeza respecto a la veracidad de dicha experiencia. Por lo tanto, ambos experimentos demuestran cómo una creencia autobiográfica falsa puede llegar a ser lo suficientemente fuerte como para generar recuerdos falsos a pesar de que no sean contextualmente verosímiles.

Un estudio similar fue realizado por Sherman et al. (2015), donde se exploró cómo las creencias falsas se podían generar mediante el paradigma Deese-Roediger-McDermot (DRM). DRM ha sido utilizado para inducir creencias falsas en términos de palabras, imágenes e inclusive nombres de marcas comerciales (Gallo, 2006). El procedimiento implica la presentación de palabras semánticamente relacionadas durante la codificación (e. g., enfermera, hospital, jeringa, etc.), las cuales luego deben de ser evocadas por los sujetos de experimentación (Pardilla-Delgado \& Payne, 2017). En el estudio de Sherman et al., se encontró que los sujetos de estudio generaron recuerdos falsos acerca de marcas comerciales, cuyo efecto era incremental conforme al paso del tiempo.

Existe también evidencia sobre memorias falsas desde la neuropsicología, al demostrarse que tanto la imaginería visual como la percepción visual dependen de los mismos mecanismos de control de áreas frontales (Dennis, Bowman, \& Turney, 2015; Ganis, Thompson, \& Kosslyn, 2004; Jeye, Karanian, \& Slotnick, 2017). Asimismo, se ha comprobado que, durante la construcción de eventos imaginarios y la recuperación de eventos reales e imaginarios, se activa una red neuronal que abarca también estructuras asociadas con la memoria autobiográfica como lo son el hipocampo, el giro hipocampal, la corteza restrosplénica, la corteza parietal posterior y la corteza prefrontal ventromedial (Hassabis, Kumaran, \& Maguire, 2007).

Por lo tanto, resulta plausible considerar que, ante la presencia de errores de distorsión en la memoria autobiográfica, el individuo lleva a cabo un proceso de construcción de la escena donde se da la generación, mantenimiento y visualización de contextos espaciales complejos subyacentes tanto a la evocación de experiencias reales como también a la imaginación de experiencias ficticias (Hassabis et al., 2007).

Todo esto nos indica que la memoria, en efecto, es proclive a sufrir un error de sugestión, ya que cuando el cerebro incorpora una creencia dentro de su esquema de procesamiento cognitivo, no se llega a discriminar entre lo real y lo imaginario puesto que se activan los mismos circuitos neuronales y se busca dar un cuerpo de sentido explicativo a la ocurrencia de experiencias relacionadas con dicha experiencia. Probablemente, este aspecto se va consolidando conforme aumenta la repetición del recuerdo falso y se posiciona con el mismo grado de detalle que una memoria verdadera.

\section{¿Pueden ser las memorias falsas adaptativas?}

Newman y Lindsay (2009) sugieren que la capacidad de generar memorias falsas se debe a que adaptativamente se ha desarrollado un mecanismo flexible que nos permite generar memorias ilusorias para imaginar el futuro $y$ reconsiderar nuestro pasado. De esta manera, se ha desarrollado la posibilidad de viajar imaginariamente hacia atrás y hacia adelante en el tiempo, con el fin de posicionar al sí mismo en un continuo de espacio y tiempo y a la vez dirigir las conductas y pensamientos con base en ello.

Este mecanismo combina tanto elementos episódicos como semánticos para recombinar y reconstruir la memoria autobiográfica en lugar de recuperar información intacta, puesto que los eventos de la vida real rara vez ocurren como una réplica directa de eventos previos y resulta necesario que exista un procesamiento flexible para adecuarse a las distintas experiencias. A 
partir de esto, es que se plantea que las memorias falsas surgen como un producto de dicho sistema adaptativo, el cual resulta proclive a sufrir de distorsiones mnémicas (i. e., sugestión).

Ambos autores plantean a su vez que las memorias falsas en sujetos sanos pueden inclusive llegar a ser adaptativas, al ser de utilidad para las necesidades del sí mismo. Las personas tienden a distorsionar ciertos aspectos de su pasado, lo cual puede servir para la construcción del sí mismo. Como evidencia de lo anterior, están las investigaciones de Sutin y Robbins (2008) y Libby y Eibach (2002), donde se halló que las personas presentan distintas perspectivas visuales de sus recuerdos autobiográficos en función de un proceso autoevaluativo. Así, cuando se recuerda a un sí mismo que se encuentra alineado con los pensamientos, sentimientos y metas actuales, la perspectiva va a ser en primera persona, caso contrario va a ser en tercera persona, puesto que es un sí mismo disonante con el sí mismo actual (i. e., "antes era otra persona").

Retomando la propensión mencionada por Schacter (2001), se comprende que las personas tienden a modificar sus recuerdos basados en creencias, expectativas y motivaciones actuales, con el propósito de brindar congruencia al sentido de identidad. De esta manera, distorsiones del pasado pueden ayudar al individuo a solventar cualquier confusión respecto a su identidad, puesto que se logran amoldar de acuerdo con la autopercepción actual, brindando una mayor cohesión en la construcción de la identidad. Es decir, si la persona cree que en un determinado evento su desempeño fue mejor que como sucedió en realidad, eso le permite mejorar su autopercepción al fortalecer su visión positiva del sí mismo. En el caso contrario de que el sujeto considere que su desempeño fue peor de como realmente sucedió, seguirá siendo adaptativo en el grado de que le motive a modificar patrones cognitivos inefectivos.

Newman y Lindsay (2009) también proponen que las memorias falsas pueden tener un rol adaptativo en la interacción social. Las distorsiones producto de una situación social pueden facilitar una evaluación más positiva por parte de un grupo hacia el individuo, al adaptarse a las creencias, expectativas y motivaciones sociales actuales y, por ende, compartir un sentido de realidad con los demás. Como ejemplo de ello, se cita el estudio de Lewandowsky, Stritzke, Oberauer y Morales (2005), donde soldados estadounidenses recordaron la existencia de armas de destrucción masiva en Irak, a pesar de ser un hecho que nunca sucedió, probablemente con el propósito de justificar sus acciones durante la guerra y evitar una exclusión social.

Asimismo, diversos autores han establecido que los recuerdos falsos son adaptativos tanto para niños como para adultos, al facilitar la resolución de problemas que requieren de soluciones basadas en introspección (Howe, 2011; Howe, Garner, Charlesworth, \& Knott, 2011; Howe, Wilkinson, Garner, \& Ball, 2016). De esta manera, ante la falta de información, la presencia de respuestas intuitivas puede fungir como una base para posteriores razonamientos que sean exitosos.

\section{Discusión}

Esencialmente, podría plantearse que toda memoria es falsa hasta cierto punto o en cierto grado (son expresiones idiomáticas ya existentes), ya que involucra un proceso inherentemente reconstructivo en el que las experiencias y eventos del pasado se integran para conformar una narrativa coherente que se convierte en nuestra autobiografía (Bernstein \& Loftus, 2009; Eisold, 2013). La existencia de memorias falsas va más allá de un cambio en el recuento de los eventos del pasado e implica una reconstrucción del recuerdo a partir de una nueva creencia que utiliza la imaginación como mecanismo de acción.

De acuerdo con Conway y PleydellPearce (2000), la construcción del recuerdo autobiográfico involucra la interacción entre una base de conocimiento y un mecanismo de control ejecutivo; sin embargo, existen otros factores que intervienen durante el proceso de construcción y recuperación del recuerdo autobiográfico. En 
primer lugar, respecto al mecanismo de control ejecutivo, Williams et al. (2007) proponen que este presenta una capacidad limitada, lo cual desemboca en posibles sesgos del funcionamiento ejecutivo contemplados bajo un reducido control atencional, control inhibitorio o memoria de trabajo, que también pueden llegar a imposibilitar una evocación isomorfa al recuerdo original.

Por otra parte, más allá de la capacidad cognoscitiva del organismo, existen variables ambientales que modulan la generación del recuerdo autobiográfico. La memoria autobiográfica es un proceso dinámico (BeltránJaimes, Moreno-López, Polo-Díaz, ZapataZabala, \& Acosta-Barreto, 2012; Conway, Pleydell-Pearce, \& Whitecross, 2001; Sutin, 2008), donde la base de conocimiento se encuentra en constante adaptación a las necesidades del sí mismo, las cuales a su vez varían en función del ambiente en que se desenvuelva el sujeto.

Lo anterior resulta plausible desde la perspectiva bio-psico-social de Welzer y Markowitsch (2005), quienes argumentan que existe una interdependencia entre las predisposiciones genéticas y el ambiente que va a determinar la maduración de la memoria autobiográfica. Bajo esta perspectiva, queda obsoleta la noción de que el desarrollo cerebral va a ser el único determinante de la capacidad mnémica, puesto que la maduración de las funciones ejecutivas, el lenguaje y la concepción de un sí mismo se dan paralelamente al desarrollo de mecanismos de interacción sociocultural.

De esta manera, durante la percepción, codificación, almacenamiento y reconstrucción de un evento se da una evaluación emocional del sí mismo que determina que tan relevante es o no para ser incorporado dentro de la narrativa de la historia de vida del sujeto y, a su vez, dicha valoración emocional se va a ver influenciada por lo establecido en el contexto sociocultural como adaptativo. Ejemplo de lo anterior, ocurre cuando las personas omiten ciertos recuerdos o detalles de la historia de vida que no concuerdan con las exigencias socioculturales ante un fenómeno de deseabilidad social.
Tomando como base lo anterior, hasta podría considerarse como adaptativo el que la memoria tenga esos "pecados de imprecisión", puesto que permiten cierta plasticidad a la hora de generar recuerdos que sean concordantes con las metas del sí mismo actual. Este aspecto junto con los aportes de Newman y Lindsay (2009), Howe (2011) y Howe et al. (2016), permite considerar que en efecto existe un rol funcionalmente adaptativo para las creencias y recuerdos falsos, y en el cual se evidencian aplicaciones a nivel bio (a partir de la evidencia de circuitos comunes de activación entre lo real e imaginario), psico (ante la evidencia del valor adaptativo para el sí mismo actual) y social (desde la evidencia del valor adaptativo sociocultural), de manera inclusivamente estratégica.

A partir de esto, sería interesante poder explorar en un futuro estudio experimental cómo factores ambientales y de desarrollo pueden afectar la aparición y mantenimiento de memorias falsas. Asimismo, poder analizar cómo se ven afectados distintos componentes de la memoria autobiográfica, puesto que esta involucra componentes episódicos, semánticos, autorreflexivos, imaginería visual, funcionamiento ejecutivo y aspectos emocionales (Adam et al., 2014; Salazar, 2008).

Otra meta u otro objetivo a considerar en una futura investigación sería el encontrar un posible correlato neurobiológico asociado con el proceso de construcción de la escena que mencionan Hassabis et al. (2007), así como la exploración de los posibles mecanismos a partir de los cuales se da la sensación de certeza de que un evento realmente sucedió, para comprender aún más el mecanismo de control cognitivo que media en la verificación de creencias. Sobre esto, se sugiere utilizar modelos formales matemáticos junto con procedimientos estadísticos (e. g., análisis de función discriminante y modelación de ecuaciones estructurales) en conjunto con el avance en nuevas técnicas de análisis conductual, emocional (e. g., análisis de la ansiedad rasgo y estado asociada) y de neuroimagen para ayudar a elucidar los procesos vinculados a la veracidad en la memoria (Beato, 
Pulido, Pinho, \& Gozalo, 2013; Bernstein \& Loftus, 2009).

A pesar de que las memorias falsas se suelen considerar como un producto de la capacidad limitada de la memoria, Newman y Lindsay (2009) nos demuestran que no existen en vano, ya que parte de su rol adaptativo consiste en ayudar al sí mismo a buscar un sentido de coherencia en relación a la conformación de su identidad y al sentido de pertenencia social.

Brindar un aporte al entendimiento de las memorias falsas no es solo relevante para analizar aspectos como la veracidad y precisión en la memoria, sino también para tratar de comprender los mecanismos de la memoria autobiográfica. Entender a la memoria autobiográfica y a la memoria en general, no como un constructo unitario sino como un sistema flexible y adaptativo, permite avanzar en su entendimiento y generar un mayor incentivo en la comunidad científica, para tratar de seguir lo propuesto por Baddeley (1988): preguntarse para qué sirve el fenómeno mnémico de estudio y así intentar descubrir la importancia de su desarrollo ontogenético.

Finalmente, cabe mencionar que, pese a que las memorias falsas no surgen a partir de eventos reales, pueden llegar a ocasionar dolor emocional similar al de personas que sí han vivido un evento traumático (Herndon, Myers, Mitchell, Kehn, \& Henry, 2014; McNally et al., 2004; Otgaar Howe, Clark, Wang, \& Merckelbach, 2015). Por lo que se deben de considerar las posibles implicaciones de los recuerdos autobiográficos falsos en ámbitos como la investigación psicológica, la práctica clínica psicológica y la psicología forense, respecto a la veracidad de la información que está siendo recolectada, sobre todo si se cuenta con evidencia que demuestra que los efectos de la sugestión de creencias falsas pueden afectar las actitudes y comportamientos de los sujetos inclusive de manera perdurable.

\section{Referencias}

Baddeley, A. (1988). But what the hell's it for? En M. M. Gruneberg, P. E. Morris \& R. N. Sykes (Eds.), Practical aspects of memory: Current research and issues, Vol. 1: Memory in everyday life (pp. 3-18). Oxford, ENG: John Wiley.

Bartlett, F. C. (1932). Remembering: A study in experimental and social psychology. Londres: Cambridge University Press.

Beato, M. S., Pulido, R. F., Pinho, M. S., \& Gozalo, M. (2013). Reconocimiento falso y ansiedad estado/rasgo. Psicológica, 34(2), 299-311. Recuperado de http://www.redaly c.org/html/169/16929535009/

Beltrán-Jaimes, J. O., Moreno-López, N. M., Polo-Díaz, J., Zapata-Zabala, M. E., \& Acosta-Barreto, M. R. (2012). Memoria autobiográfica: un sistema funcionalmente definido. International Journal of Psychological Research, 5(2), 108-123. Recuperado de https://www.researchgate.net/publicatio n/274264606_Memoria_autobiografica_un _sistema_funcionalmente_definido

Berlyne, N. (1972). Confabulation. The British Journal of Psychiatry, 120, 31-39. https://doi .org/10.1192/bjp.120.554.31

Bernstein, D. M., \& Loftus, E. F. (2009). How to tell if a particular memory is true or false. Perspectives on Psychological Science, 4, 370-374. https://doi.org/10.1111/j.1745-69 24.2009.01140.x

Blank, H. (1998). Memory states and memory tasks: An integrative framework for eyewitness memory and suggestibility. Memory, 6(5), 481-529. https://doi.org/10.1 080/741943086

Braun, K. A., Ellis, R., \& Loftus, E. F. (2002). Make my memory: How advertising can change our memories of the past. Psychology ES Marketing, 19, 1-23. Recuperado de http ://scholarship.sha.cornell.edu/articles/332

Brewin, C. R., \& Andrews, B. (2017). Creating memories for false autobiographical events in childhood: A systematic review. Applied 
Cognitive Psychology, 31, 2-23. https://doi.o rg/10.1002/acp.3220

Conway, M. A. (2005). Memory and the self. Journal of Memory and Language, 53, 594628. http://doi.org/10.1016/j.jml.2005.08.0 05

Conway, M. A., \& Loveday, C. (2014). Remembering, imagining, false memories $\&$ personal meanings. Consciousness and Cognition, 33, 574-581. https://doi.org/10.1 016/j.concog.2014.12.002

Conway, M. A. \& Pleydell-Pearce, C. W. (2000). The construction of autobiographical memories in the self-memory system. Psychological Review, 107(2), 261-288. http ://doi.org/10.1037/0033-295X.107.2.261

Conway, M. A., Pleydell-Pearce, C. W., \& Whitecross, S. E. (2001). The neuroanatomy of autobiographical memory: A slow cortical potential study of autobiographical memory retrieval. Journal of Memory and Language, 45, 493-524. http s://doi.org/10.1006/jmla.2001.2781

Dennis, N. A., Bowman, C. R., \& Turney, I. C. (2015). Functional neuroimaging of false memories. En A. Duarte, D. R. Addis \& M. Barense (Eds.), The Wiley handbook on the cognitive neuroscience of memory (pp. 150-171). Chichester, UK: Wiley-Blackwell.

Eisold, K. (17 de agosto de 2013). All memories are false. Visions of Frankenstein [Mensaje en un blog]. Recuperado de https://www.psychologytoday.com/blog/ hidden-motives/201308/all-memories-are-f alse

Finkelstein, J. D. (2017). The $\Psi$-files: A review of the psychological literature regarding false memories of alien abduction. The New School Psychology Bulletin, 14(1), 37-44. Recuperado de http://www.nspb.net/index. $\mathrm{php} / \mathrm{nspb} /$ issue/viewIssue/38/11

Gallo, D. A. (2006). Associative illusions of memory: False memory research in DRM and related tasks. Nueva York: Psychology Press.

Ganis, G., Thompson, W. L., \& Kosslyn, S. M. (2004). Brain areas underlying visual mental imagery and visual perception: An
fMRI study. Cognitive Brain Research, 20, 226-241. https://doi.org/10.1016/j.cogbrain res.2004.02.012

Garry, M., \& Gerrie, M. P. (2005). When photographs create false memories. Current Directions in Psychological Science, 14(6), 321-360. https://doi.org/10.1111/j.0963-72 14.2005.00390.x

Hassabis, D., Kumaran, D., \& Maguire, E. A. (2007). Using imagination to understand the neural basis of episodic memory. The Journal of Neuroscience, 27(52), 14365-14374. https://doi.org/10.1523/JNE UROSCI.4549-07.2007

Hering, E. (1895). Über das Gedächtnis als eine allgemeine Funktion der organisierten Materie [Memory as a general function of organized matter]. Chicago, IL: Open Court.

Herndon, P., Myers, B., Mitchell, K., Kehn, A., \& Herny, S. (2014). False memories for highly aversive early childhood events: Effects of guided imagery and group influence. Psychology of Consciousness: Theory, Research, and Practice, 1, 20-31. htt ps://doi.org/10.1037/cns0000011

Howe, M. L. (2011). The adaptive nature of memory and its illusions. Current Directions in Psychological Science, 20(5), 312-315. htt ps://doi.org/10.1177/0963721411416571

Howe, M. L., Garner, S. R., Charlesworth, M., \& Knott, L. (2011). A brighter side to memory illusions: False memories prime children's and adults' insight-based problem solving. Journal of Experimental Child Psychology, 108(2), 383-393. https://doi.org/10.1016/j.j ecp.2010.08.012

Howe, M. L., Wilkinson, S., Garner, S. R., \& Ball, L. J. (2016). On the adaptive function of children's and adults' false memories. Memory, 24(8), 1062-1077. https://doi.org/ 10.1080/09658211.2015.1068335

Jeye, B. M., Karanian, J. M., \& Slotnick, S. D. (2017). The anterior prefrontal cortex and the hippocampus are negatively correlated during false memories. Brain Sciences, 7(13), 1-9. https://doi.org/10.3390 /brainsci7010013 
Joselyne Nájera Fernández, Mónica Salazar Villanea, Jaime Fornaguera Trías.

Koriat, A., Goldsmith, M., \& Pansky, A. (2000). Toward a psychology of memory accuracy. Annual Review of Psychology, 51, 481-537. https://doi.org/10.1146/annurev. psych.51.1.481

Laney, C., Bowman-Fowler, N., Nelson, K. J., Bernstein, D. M., \& Loftus, E. F. (2008). The persistence of false beliefs. Acta Psychologica, 129, 190-197. https://doi.org/1 0.1016/j.actpsy.2008.05.010

Laney, C., Morris, E. K., Bernstein, D. M., Wakefield, B. M., \& Loftus, E. F. (2008). Asparagus, a love story: Healthier eating could be just a false memory away. Experimental Psychology, 55(5), 291-300. ht tps://doi.org/10.1027/1618-3169.55.5.291

Lewandowsky, S., Stritzke, W. G. K., Oberauer, K., \& Morales, M. (2005). Memory for fact, fiction, and misinformation. Psychological Science, 16(3), 190-195. https://doi.org/10. 1111/j.0956-7976.2005.00802.x

Libby, L., \& Eibach, R. (2002). Looking back in time: Self-concept change affects visual perspective in autobiographical memory. Journal of Personality and Social Psychology, 82 (2), 167-179. https://doi.org/10.1037/00 22-3514.82.2.167

Lindsay, D. S., Hagen, L., Read, J. D., Wade, K. A., \& Garry, M. (2004). True photographs and false memories. Psychological Science, 15(3), 149-154. https://doi.org/10.1111/j.0 956-7976.2004.01503002.x

Loftus, E. F., \& Davis, D. (2006). Recovered memories. Annual Review of Clinical Psychology, 2, 469-498. https://doi.org/10.1 146/annurev.clinpsy.2.022305.095315

Loftus, E. F., \& Pickrell, J. E. (1995). The formation of false memories. Psychiatric Annals, 25, 720-725. https://doi.org/10.392 8/0048-5713-19951201-07

Mazzoni, G., \& Memon, A. (2003). Imagination can create false autobiographical memories. Psychological Science, 14(2), 186-188. https://doi.org/10.1046/j.1432-13 27.1999.00020.x

McNally, R. J., Lasko, N. B., Clancy, S. A., Macklin, M. L., Pitman, R. K., \& Orr, S. P. (2004). Psychophysiological responding during script driven imagery in people reporting abduction by space aliens. Psychological Science, 15(7), 493-497. https://doi.org/10.1111/j.0956-79 76.2004.00707.x

Mendez, M. F., \& Fras, I. A. (2011). The false memory syndrome: Experimental studies and comparison to confabulations. Medical Hypotheses, 76, 492-496. https://doi.org/10. 1016/j.mehy.2010.11.033

Nelson, K. (1993). The psychological and social origins of autobiographical memory. Psychological Science, 4, 7-14. https://doi.org /10.1111/j.1467-9280.1993.tb00548.x

Newman, E. J., \& Lindsay, D. S. (2009). False memories: What the hell are they for? Applied Cognitive Psychology, 23, 1105-1121. https://doi.org/10.1002/acp.1613

Otgaar, H., Howe, M. L., Clark, A., Wang, J., \& Merckelbach, H. (2015). What if you went to the police and accused your uncle of abuse? Misunderstandings concerning the benefits of memory distortion: A commentary on Fernandez (2015). Consciousness and Cognition, 33, 286-290. https://doi.org/10.1016/j.concog. 2015.01.015

Pardilla-Delgado, E., \& Payne, J. D. (2017). The Deese-Roediger-McDermott (DRM) task: A simple cognitive paradigm to investigate false memories in the laboratory. Journal of Visualized Experiments, 119, e54793. https:// doi.org/10.3791/54793

Prebble, S. C. (2014). Autobiographical memory and sense of self (Tesis doctoral inédita). Universidad de Auckland, Nueva Zelanda.

Salazar, M. (2008). Estudio de las características de la memoria autobiográfica en la vejez: la reminiscencia en sujetos sin deterioro cognitivo $y$ en sujetos con demencia tipo Alzheimer (Tesis doctoral inédita). Universidad de Salamanca, España.

Schacter, D. L. (1996). Searching for memory: The brain, the mind, and the past. Nueva York: Basic Books.

Schacter, D. L. (2001). The seven sins of memory: How the mind forgets and remembers. Boston, MA: Houghton, Mifflin \& Company. 
Schacter, D. L., Chiao, J. Y., \& Mitchell, J. (2003). The seven sins of memroy: Implications for the self. Annals of the New York Academy of Sciences, 1001, 226-239. https://doi.org/10. 1196/annals.1279.012

Schacter, D. L., Guerin, S. A., \& St. Jacques, P. L. (2011). Memory distortion: An adaptive perspective. Trends in Cognitive Sciences, 15, 467-474. https://doi.org/10.1016/j.tics.2 011.08 .004

Sherman, S. M., Follows, H., Mushore, A. B., Hampson-Jones, K., \& Wright-Bevans, K. (2015). Television advertisements create false memories for competitor brands. Journal of Applied Research in Memory and Cognition, 4, 1-7. https://doi.org/10.1016/j.j armac.2014.06.001

Staniloiu, A., \& Markowitsch, H. J. (2012). Dissociation, memory and trauma narrative. Journal of Literary Theory, 6, 103-130. https://doi.org/10.1515/jlt-2011-0 012

Sutin, A. R. (2008). Autobiographical memory as a dynamic process: Autobiographical memory mediates basic tendencies and characteristic adaptations. Journal of Research in Personality, 42 (2), 1060-1066. h ttps://doi.org/10.1016/j.jrp.2007.10.002

Sutin, A., \& Robins, R. (2008). When the "I" looks at the "me": Autobiographical memory, visual perspective, and the self. Consciousness and Cognition, 17(4), 1386-1397. https://doi.org/10.1016/j.conco g.2008.09.001

Tulving, E. (1972). Episodic and semantic memory. En E. Tulving \& W. Donaldson (Eds.), Organization of memory (pp. 381-403). Nueva York: Academic Press.

Tulving, E., \& Markowitsch, H. J. (1998). Episodic and declarative memory: Role of the hippocampus. Hippocampus, 8(3), 198-204. https://doi.org/10.1002/ (SICI) 1098-1063(1998) 8:3<198::AID$\mathrm{HIPO} 2>3.0 . \mathrm{CO} ; 2-\mathrm{G}$

Wade, K. A., Garry, M., Read, J. D., \& Lindsay, D. S. (2002). A picture is worth a thousand lies: Using false photographs to create false childhood memories. Psychonomic Bulletin and Review, 9(3), 597-603. https://doi.org/1 $0.3758 / \mathrm{BF} 03196318$

Welzer, H., \& Markowitsch, H. (2005). Towards a bio-psycho-social model of autobiographical memory. Memory, 13(1), 63-78. https://doi.org/10.1080/0965821034 4000576

Williams, J. M. G., Barnhofer, T., Crane, C., Herman, D., Raes, F., Watkins, E., \& Dalgleish, T. (2007). Autobiographical memory specificity and emotional disorder. Psychological Bulletin, 33, 122-148. https://d oi.org/10.1037/0033-2909.133.1.122

Wilson, A., \& Ross, M. (2003). The identity function of autobiographical memory: Time is on our side. Memory, 11, 137-149. https: //doi.org/10.1080/741938210

\section{Notas}

* Artículo de investigación.

1 La memoria episódica permite el recuerdo consciente de hechos o eventos del pasado experimentados personalmente, mientras que la memoria semántica es la encargada de la adquisición, retención y recuperación de la información relativa al mundo (Conway \& Loveday, 2014; Prebble, 2014; Tulving, 1972). 